\title{
Original
}

\section{Asociación entre anticuerpos antifosfolípidos y complicaciones de la gestación en mujeres de Costa Rica}

\author{
Roubier Rojas-Barahona', Juan José Romero-Zúñiga', Lizbeth Salazar-Sánchez²
}

\section{Resumen}

Objetivo: Determinar asociación entre los anticuerpos antifosfolípidos y complicaciones de la gestación (aborto de cualquier tipo, preeclampsia, eclampsia, insuficiencia placentaria grave o parto antes de la semana 34 de gestación), tomando como base la población de pacientes del servicio de Obstetricia del Hospital México.

Materiales y métodos: Estudio de casos y controles en la población de pacientes atendidas por el servicio de Obstetricia del Hospital México en el período comprendido entre enero de 2002 y diciembre de 2004. Se tomaron los datos del registro de egresos Hospitalarios, se hizo un análisis descriptivo, y un análisis univariado y multivariado de las principales variables registradas.

Resultados: El promedio de edad de las pacientes en los casos y los controles no mostró diferencia significativa. La provincia de San José reporta el mayor porcentaje de casos con un $39.9 \%$, seguido por Heredia con un $26.8 \%$ y Alajuela con un $24.6 \%$. En cuanto al número de gestaciones, se determinó que no influyen significativamente en relación con el aborto y otras complicaciones de la gestación. El análisis univariado y multivariado mantiene asociación entre los antecedentes clínicos de las gestaciones anteriores, además de los anticuerpos antifosfolípidos

I Posgrado Regional en Ciencias Veterinarias Tropicales. Universidad Nacional

2 Centro de Investigación en Hemoglobinas Anormales y Trastornos Afines de la Universidad de Costa Rica (CIHATA). Universidad de Costa Rica

Abreviaturas: $\quad \mathrm{aFL}$, anticuerpos antifosfolípidos; $\mathrm{aCL}, \quad$ anticuerpos anticardiolipina $\lg \mathrm{g}$ e $\lg M$; $\mathrm{AL}$, anticoagulante lúpico; $\mathrm{DE}$, desviación estándar; IC 95\%, Intervalo confianza al 95\%; SAF, Síndrome antifofolípido.

Correspondencia: Juan José Romero Zúñiga Apdo.304-3000 Heredia e-mailjromero@medvet. una.ac.cr

ISSN 0001-6002/2007/49/2/102-106 Acta Médica Costarricense, $\mathbb{C} 2007$ Colegio de Médicos y Cirujanos respecto al aborto y otras complicaciones de la gestación.

Conclusión: Los anticuerpos antifosfolípidos deberían ser tomados en cuenta como parte del seguimiento del embarazo en pacientes con historia de abortos y complicaciones de la gestación en embarazos previos.

Descriptores: aborto, complicaciones de la gestación, anticuerpos antifosfolípidos, estudio caso-control, Costa Rica.

Keywords: abortion, pregnancy failures, antiphospholipid antibodies, case-control study, Costa Rica.

Recibido: 28 de agosto de 2006

Aceptado: 8 de febrero de 2007

El síndrome antifosfolípido (SAF) ha sido definido como un estado de trombofilia o abortos a repetición, asociado a la presencia de anticuerpos antifosfolípidos (aFL) a título moderado o alto y acompañado con cierta frecuencia de trombocitopenia. ${ }^{1}$ Se cataloga como una enfermedad autoinmune que cursa con manifestaciones clínicas de trombosis arterial o venosa y abortos recurrentes, ${ }^{2}$ y que puede presentarse en pacientes con otras enfermedades del tejido conectivo, particularmente el lupus eritematoso sistemático (LES) y la artritis reumatoide, denominándose, en estos casos, SAF secundario, y primario si no se asocia a ninguna otra enfermedad. ${ }^{3}$ Asimismo, ocurre con mayor frecuencia en los adultos jóvenes y existe un predominio en la población femenina. ${ }^{4}$

Los fosfolípidos son una familia heterogénea de inmunoglobulinas cuya especificidad se ha definido experimentalmente. Se ha demostrado que estos anticuerpos presentan distinta afinidad 
hacia diversas proteínas asociadas a fenómenos trombóticos, dentro de los cuales existen dos grupos de anticuerpos reconocidos, los anticuerpos anticardiolipinas $(\mathrm{aCL})$ y el anticoagulante lúpico $(\mathrm{AL})$. Existe una concordancia parcial entre los aFL y se estima que el $80 \%$ de los pacientes con $\mathrm{AL}$ tienen aCL y menos del $50 \%$ de los pacientes con aCL presenta AL. ${ }^{5}$ Por ello es importante la realización de análisis conjuntos tanto de aCL como de AL.

Algunas estimaciones hablan de prevalencias de entre el $1-8 \%$ de aCL y AL; sin embargo, los trabajos sobre riesgo trombótico asociado y complicaciones en el embarazo son contradictorios. ${ }^{6}$ Otros autores han observado que existe un mayor riesgo cuando el título de aFL aumenta, lo que favorece la probabilidad de desarrollar SAF. ${ }^{7}$

El 82\% de los pacientes con SAF son mujeres, en una relación de 5:1 respecto a los varones, diferencia que se acentúa cuando se trata de SAF secundario, ${ }^{8}$ y son diagnosticadas, en un $85 \%$ de los casos, entre los 15 y los 50 años. ${ }^{9}$ Asimismo, la presencia de aFL puede afectar los factores tradicionales de riesgo, como la trombosis venosa ó arterial, el embarazo y los procedimientos quirúrgicos. ${ }^{10}$

El aborto recurrente se asocia con la presencia de aFL, producen complicaciones durante el embarazo debido a trombosis de los vasos placentarios, infarto e insuficiencia placentaria. Como resultado, se podría ocasionar retardo en el crecimiento intrauterino y la muerte fetal posterior. ${ }^{11} \mathrm{De}$ allí la importancia de la determinación de aFL antes del embarazo o durante las primeras semanas de gestación, en las pacientes con pérdidas fetales recurrentes. ${ }^{12}$

Se sabe que el SAF está asociado con entre un 7\% y un $48 \%$ de las pérdidas fetales, pérdidas preembrionarias hasta la cuarta semana de gestación y embrionarias de la semana 5 a la 9 de gestación. ${ }^{13}$ Las pérdidas asociadas al SAF suelen ocurrir en el segundo y tercer trimestre del embarazo y tener causa posiblemente inmunológica, mientras que en la población normal las pérdidas ocurren durante el primer trimestre del embarazo y se relacionan con causas no inmunológicas, como las alteraciones morfológicas o cromosómicas. ${ }^{14}$ La historia de los embarazos previos es importante para determinar la probabilidad de una prueba positiva para $\mathrm{AL}$ y aCL. ${ }^{15}$

En el Hospital México se presentan anualmente cerca de 500 abortos y otros casos de problemas de la gestación, que quizás se deban a la presencia de aFL, y por criterio clínico correspondan a SAF, lo cual es importante de conocer por las implicaciones sociales y económicas para la sociedad, el núcleo familiar y la paciente. Por tanto, el objetivo del estudio es determinar asociación entre los aFL y las complicaciones de la gestación en Costa Rica, tomando como referencia el servicio de Obstetricia del Hospital México, de enero de 2002 a diciembre de 2004.

\section{Materiales y métodos}

La población estudiada comprendió a las mujeres en edad fértil (desde los 11 hasta los 50 años) que consultaron en el servicio de Obstetricia del Hospital México, durante el período del 1 de enero de 2002 al 31 de diciembre de 2004 . Se incluyeron aquellas a las quienes se les realizó la determinación de aFL en el lapso de 6 meses antes o después del embarazo atendido durante el período de estudio. No hubo distingo por edad, grupo étnico o zona geográfica.

Se consideró como "caso" a aquellas pacientes del servicio que durante la gestación analizada: 1) sufrieran un aborto de cualquier tipo, 2) padecieran complicaciones en la gestación como preeclampsia, eclampsia o insuficiencia placentaria grave, ó 3) presentaran partos antes de la semana 34 de gestación. Por otra parte, se tomó como "control" a las pacientes con parto normal a término, atendidas en el mismo hospital dentro del período de estudio.

Se utilizó un diseño de casos y controles a una razón mínima de 1:1. El tamaño de la muestra se obtuvo por medio de la fórmula sugerida por Rothman (1986) ${ }^{16}$ Según esto, se requería de un mínimo de 80 pacientes en cada grupo; sin embargo, fue posible colectar datos de 138 casos y 271 controles, para una relación de 1 caso por casi 2 controles, con un incremento del poder del estudio.

Los datos corresponden a los registros de egresos hospitalarios por aborto y complicaciones en la gestación en el Hospital México facilitados por el Departamento de Bioestadística. Otros antecedentes, se obtuvieron del expediente clínico existente en el archivo del Hospital. Se utilizó los resultados cuantitativos de análisis de AL del registro del laboratorio de Hematología y los de aCL de los registros del laboratorio de Inmunología. El presente estudio fue aprobado por el Comité Ético Científico y la Dirección del Hospital México, de la Caja Costarricense de Seguro Social.

Se revisaron los datos para evitar errores de digitación o perdidas. Las variables continuas se convirtieron a categóricas y se codificaron de manera creciente con respecto el riesgo, siguiendo lo reportado en la bibliografía. Se clasificó como positiva la presencia de aFL en aquellas pacientes con la prueba de neutralización mediante fosfolípidos purificados de fase hexagonal confirmatoria de AL positiva (diferencia mayor de 8 segundos). También fueron positivas las que presentaron aCL positiva para $\mathrm{IgG} \mathrm{o}$ IgM (mayor o igual de $20 \mathrm{U} / \mathrm{ml}$ ).

Se creó una base de datos en Epi Info 2002 (Centers for Diseases Control and Prevention, 2002). ${ }^{17}$-Se eliminaron los datos extremos o no plausibles, mediante análisis por estadística descriptiva, pruebas de normalidad y de tendencia. 
En cuanto al análisis de los datos, se realizó la descripción de las pacientes mediante el uso de las medidas de frecuencias con sus respectivos intervalos de confianza para cada estrato, y medidas de tendencia central y de dispersión.

Se efectuó un análisis univariado por medio de tablas de $2 \mathrm{x} \mathrm{k}$ para determinar la magnitud y la dirección de la asociación entre el evento aborto o complicaciones de la gestación y cada variable, por medio de la razones de posibilidades (Odds Ratio). Adicionalmente, se calculó las fracciones atribuibles.

En el análisis multivariado se incluyeron las variables que al análisis univariado tuvieron valores de $\mathrm{p} \leq 0.25$. Se siguió una estrategia de modelaje paso a paso en reversa, basado en el valor de p de la prueba de razón de verosimilitud. ${ }^{18}$ Ambos análisis (univariado y multivariado) se efectuaron utilizando el programa EGRET®.

\section{Resultados}

En el análisis descriptivo no existió diferencia en la media de los valores de anticoagulante lúdico entre casos y controles, siendo 4.5 y 3.5 segundos, respectivamente. Del mismo modo, no existe diferencia en la media de los anticuerpos anticardiolipinas IgG, que para los casos fue de $12.5 \mathrm{U} / \mathrm{ml}$ y en los controles $13.0 \mathrm{U} / \mathrm{ml}$, como se observa en el Cuadro 1.

En el análisis univariado (Cuadro 2) se evidenció que la edad (categorizada) y el número de gestaciones no influyen de forma significativa sobre el aborto $\mathrm{u}$ otras complicaciones del embarazo. Por el contrario, los antecedentes de gestaciones anteriores y la presencia de anticuerpos antifosfolípidos (AL o aCL positivos) evidenciaron una estrecha asociación con el aborto y otras complicaciones de la gestación(OR: 38.5, IC95\% 14.2-113.3). Adicionalmente, al evaluar por separado los anticuerpos anticardiolipinas (IgG e IgM), se determinó que los IgG presentaron fuerte asociación (OR: 14.6, IC95\% 3.9 - 64.2, p < 0.01), mientras que los IgM indicaron una débil asociación (OR: 3.3, IC95\%
$0.8-16.2)$ con el aborto y otras complicaciones de la gestación.

La fracción atribuible de los abortos o complicaciones en la gestación por la presencia de aFL fue del $40 \%$, lo que indica que esta proporción de casos podría ser prevenida si los aFL no estuvieran presentes.

Con el análisis multivariado se reafirmaron los hallazgos del análisis univariado, de modo que tanto la edad como el número de gestaciones de la paciente no resultan factores de riesgo para los abortos y otras complicaciones de la gestación, mientras que la presencia de anticuerpos antifosfolípidos y los antecedentes clínicos de la paciente en gestaciones anteriores sí son factores de riesgo para los abortos y otras complicaciones, con OR de 33.3 (IC95, 10.7-103.0) y 45.2 (IC95\%, 5.5-373.4), respectivamente (Cuadro 3).

\section{Discusión}

El aborto y las complicaciones de la gestación son condiciones que generan efectos permanentes en las madres y en los núcleos familiares. El conocimiento de los factores que pueden conducir a este tipo de desenlace, casi desde antes de la misma concepción, pueden colaborar a reducir el número de estos eventos y sus efectos colaterales, no solo para la paciente y su familia sino para el sistema de salud en general.

Esta investigación determinó algunos factores de riesgo para el aborto y otras complicaciones de la gestación en la población costarricense. Si bien es cierto que no se estudió el SAF debido a la falta de los datos clínicos, los resultados son reveladores en el tanto que se pudo demostrar que la presencia de aFL en títulos moderados y altos presentó una alta asociación (riesgo) con el aborto y otras complicaciones de la gestación, tanto en el caso del AL como al evaluar por separado la determinación de aCL de tipo IgG, y del tipo IgM. La presencia de aFL a título moderado o alto, como parte del SAF, se ha asociado con abortos recurrentes. ${ }^{19}$ Diversos estudios relacionan los aCL del isotipo IgG (prueba

\begin{tabular}{|c|c|c|c|c|c|c|}
\hline \multicolumn{7}{|c|}{$\begin{array}{l}\text { Cuadro 1. Niveles de aFL en las pacientes (casos y controles) del Servicio de Obstetricia del Hospita } \\
\text { México, enero de } 2002 \text { a diciembre de } 2004\end{array}$} \\
\hline \multirow{2}{*}{ Variable } & \multirow{2}{*}{ Nivel de variable } & \multicolumn{2}{|c|}{ Casos $(n=138)$} & \multicolumn{2}{|c|}{ Controles $(n=271)$} & \multirow{2}{*}{$\mathrm{p}^{*}$} \\
\hline & & Media (DE) & IC 95\% & Media (DE) & IC 95\% & \\
\hline \multirow[t]{3}{*}{$\mathrm{aFL}$} & AL (segundos) & $4.5(10.7)$ & $3.1-5.9$ & $3.5(6.7)$ & $3.0-4.0$ & 0.25 \\
\hline & $\mathrm{aCL}(\operatorname{lgG}, \mathrm{U} / \mathrm{ml})$ & $12.5(10.4)$ & $11.1-13.9$ & $13.0(7.7)$ & $12.4-14.0$ & 0.57 \\
\hline & $\mathrm{aCL}(\mathrm{IgM}, \mathrm{U} / \mathrm{ml})$ & $10.4(6.7)$ & $9.4-11.3$ & $10.9(6.2)$ & $10.5-11.4$ & 0.39 \\
\hline
\end{tabular}




\begin{tabular}{|lccccc|}
\hline \multicolumn{5}{|c|}{$\begin{array}{c}\text { Cuadro 2. Análisis univariado para los factores asociados al aborto y complicaciones de la } \\
\text { gestación en pacientes atendidas por el Servicio de Obstetricia del Hospital México, } \\
\text { enero de } 2002 \text { a diciembre de 2004 }\end{array}$} \\
\hline Variable & $\begin{array}{l}\text { Nivel de } \\
\text { variable }\end{array}$ & OR & IC $95 \%$ & FA exp. & p \\
\hline Edad & $19-34$ & - & - & - & - \\
& $<19$ & 1.4 & $0.8-2.5$ & 0.06 & 0.18 \\
Número de gestaciones & $>35$ & 1.0 & $0.4-2.5$ & 0.04 & 0.90 \\
& $<3$ & - & - & - & - \\
Antecedentes gesta/previas & $>2$ & 0.9 & $0.6-1.5$ & 0.01 & 0.46 \\
& No & - & - & - & - \\
aCL/lgG & Sí & 46.8 & $6.3-964.1$ & 0.21 & $<0.01$ \\
& No & - & - & - & - \\
aCL/lgM & Sí & 14.6 & $3.9-64.2$ & 0.35 & $<0.01$ \\
& No & - & - & - & - \\
AL & Sí & 3.3 & $0.8-16.3$ & 0.08 & $<0.06$ \\
& No & - & - & - & - \\
aFL & Sí & 47.0 & $10.4-294.3$ & 0.31 & $<0.01$ \\
& No & - & - & - & - \\
& Sí & 38.5 & $14.2-113.3$ & 0.40 & $<0.01$ \\
\hline
\end{tabular}

\begin{tabular}{|lrcc|}
\hline \multicolumn{4}{|c|}{ Cuadro 3. Análisis multivariado para los } \\
factores asociados al aborto y complicaciones \\
de la gestación en pacientes atendidas por el \\
$\begin{array}{c}\text { Servicio de Obstetricia del Hospital México, } \\
\text { enero de 2002 a diciembre de 2004 }\end{array}$ \\
\hline Variable & OR & IC 95\% & p \\
\hline Edad & 1.3 & $0.7-2.4$ & 0.30 \\
Número de gestaciones & 1.1 & $0.7-1.9$ & 0.61 \\
Antecedentes & 45.2 & $5.5-373.4$ & $<0.01$ \\
aFL & 33.3 & $10.7-103.0$ & $<0.01$ \\
\hline
\end{tabular}

de mayor sensibilidad) y el AL (prueba de mayor especificidad), por lo que resulta relevante su determinación antes, durante y después del embarazo. ${ }^{20}$ Este dato es importante desde el punto de vista de la medicina preventiva, pues si se identifica la presencia de estos anticuerpos en las pacientes embarazadas y se les proporciona el seguimiento y tratamiento adecuado, se podría prevenir una alta proporción de abortos o complicaciones de la gestación. ${ }^{19,21}$

En este estudio, los antecedentes clínicos de gestaciones anteriores se asociaron significativamente con abortos y otras complicaciones de la gestación, lo cual concuerda con los resultados de García-Campos et al (1995), que indican que el conocimiento de los antecedentes clínicos de las pacientes podría ser un indicador que contribuya a disminuir el riesgo de abortos y complicaciones de la gestación., ${ }^{2,15,22}$ Asimismo, conociendo este tipo de antecedentes, sería posible prevenir hasta un $21 \%$ de los abortos y otras complicaciones de la gestación. . $^{15,22}$

A la luz de los resultados de este estudio, es posible concluir que la determinación de aFL antes o durante la gestación, principalmente cuando existan antecedentes clínicos de importancia en gestaciones previas sugestivos de SAF, podría beneficiar la reducción de los abortos y las complicaciones de la gestación en la población de mujeres costarricenses. Es el parecer de los investigadores, que deben realizarse mayores esfuerzos por diagnosticar el SAF en etapas tempranas de la gestación

Esta investigación aporta mucho al elucidar algunas de las causas de abortos o complicaciones de la gestación en mujeres costarricenses, pues al concordar en resultados y conclusiones con otros estudios efectuadas en distintas latitudes, reafirma la necesidad de poner mayor atención a los aFL (y al SAF de ser posible), e insta a continuar posteriores investigaciones locales acerca del tema. 


\section{Abstract}

Aim: To determine the association between antiphospholipid antibodies and pregnancy failures (abortion, preclampsia, eclampsia, placental insufficiency or delivery before the $34^{\text {th }}$ week of gestation) in Costa Rican women.

Materials and Methods: The present is a case-control study, the participants belong to the Obstetrics Department of the Hospital Mexico. The period of the study was from January 2002 to December 2004. The data was obtained from the discharge registry of the Hospital descriptive analysis was done followed by univariate and multivariate analysis of the main variables.

Results: The age the patients did not show difference between cases and controls. San Jose reported a higher percentage of cases with $39.9 \%$, followed by Heredia $(26.8 \%)$ and Alajuela (24.6\%). An association between the antiphospholipid antibodies and pregnancy failures in women in Costa Rica was found. The number of pregnancies did not affect the abortion and other pregnancy failures. With the unvaried and multivariate analyses a strong association between the history of the previous pregnancies was determined, and a high relationship between the antiphospholipid antibodies with abortion and other pregnancy failures was also found.

Conclusion: The antiphospholipid antibodies should be taken into account during the follow-up of the pregnancy in patients with history of abortions or pregnancy failures.

\section{Referencias}

1. Roubey RA. Immunology of the antiphospholipid antibody syndrome. Arthritis Rheum 1996; 39:1444-1454.

2. Campos-García MT, Guil-García M, Grana Ml, Garrido-de Ramón E. Manifestaciones clínicas y analíticas del síndrome antifosfolípido primario. Med Clin1995; 104:37-38.

3. Levine JS, Branch, Rauch J. The antiphospholipid syndrome. Engl J Med 2002; 346: 752-763.

4. Alarcón-Segovia, D, Cabral AR. The concept and classification of antiphospholipid /cofactor syndromes. Lupus 1996; 5:364-367.
5. Orts JA, Zúñiga A, Orera M. Actualización de síndrome antifosfolípido. Med Clin (Barc) 2003; 121:459-471.

6. Petri M. Epidemiology of the antiphospholipid antibody. J Autoimmun 2000; 15:145-151.

7. Harris E, Pierangelli SS. Revisiting the anticardiolipin test and its standardization. Lupus 2002; 11:269-275.

8. Ware D, Branch, Munther A, Khamashta. Antiphospholipid Syndrome: Obstetric Diagnosis, Management, and Controversies. Obstetric Gynecol 2003; 101:1333-13344.

9. Cervera R, Piette JC, Font J, Khamastha MA, Shoenfeld Y, Campos MT. Antiphospholipid syndrome: clinical and immunologic manifestations and patterns of disease expression in a cohort of 1000 patients. Arthritis Rheum 2002; 46:1019-1027.

10. Erkan D, Yazici Y, Sobel R, Lockshin MD. Primary antiphopholipid syndrome: functional outcomes alter 10 years. J Rheumatol 2000; 27:2817-2821.

11. Chi HS. Recent advances in the diagnosis of antiphopholipid syndrome. Int J Hematol 2002; 76 (Suppl 2): 47-51.

12. Khamastha M.A. Hughes syndrome: Antiphospholipid syndrome. London: Springer Publishing Company, 2000.

13. Brandt JT, Triplett DA, Alving B, Scharrer I. Criteria for the diagnosis of lupus anticoagulants: an update. Thromb Haemost 1995; 74:11851190.

14. Muñoz-Rodríguez FJ, Reverter JC, Font J, Tassies D, Espinoza G, Cervera R. Clinical significance of acquired active protein $\mathrm{C}$ resistance in patients with systemic lupus erythematosus. Lupus 2002; 11: 641643.

15. Sebire NJ, Regan L, Rai R. Biology and pathology of the placenta in regulation to antiphospholipid antibody- associated pregnancy failure. Lupus 2002; 11:641-643.

16. Rothman JK. Modern epidemiology. Boston: Little, Brown \& Co., 1986.

17. Centers for Diseases Control and Prevention. Epi Info 2002, Manual for users. Atlanta: CDC, 2002.

18. Hosmer DW, Lemeshow S. Applied logistic regression, textbook and solutions manual. Chichester: Willey \& Sons, 1989.

19. Sefer G. Antiphospholipidid Syndrome. Dismounts 2003; 49:691742.

20. Pierangelli SS, Gharavi AE, Harris E. Testing for antiphopholipid antibodies: problems and solutions. Clin Obstet Gynecol 2001; 44:4857.

21. Carbone J, Sarmiento E, Segovia P, Rodríguez-Mahon M., RodríguezMolina JJ., Fernández-Cruz E. Anticuerpos antifosfolípidos y evolución a conectivopatía: estudio de seguimiento de mujeres con abortos recurrentes. Med Clin 2002; 119:681-685.

22. Erkan D. The relation between antiphospholipid syndrome-related pregnancy morbidity and non-gravid vascular thrombosis: a review of literature and management strategies. Curr Rheumatol Rep 2002; 4:379-386. 